\title{
1 Immunological role of primary cilia of dendritic cells in human skin disease
}

2 Manami Toriyama ${ }^{1,2,3, *}$, Defri Rizaldy ${ }^{1,2,4}$ Motoki Nakamura ${ }^{5}$, Fumitaka Fujita ${ }^{1,2,6}$, Fumihiro

$$
\text { Okada }^{1,6} \text {, Akimichi Morita }{ }^{5}, \text { Ken J. Ishii }^{2,7,8^{*}}
$$

4 1. Graduate School of Pharmacological Sciences, Osaka University, Osaka, Japan

5 2. Center for Vaccine and Adjuvant Research (CVAR), National Institutes of Biomedical

6 Innovation, Health and Nutrition, Osaka, Japan

7 3. Graduate School of Science and Technology, Nara Institute of Science and Technology,

8 Nara, Japan

9 4. School of Pharmacy, Institut Teknologi Bandung, Indonesia

10 5. Department of Geriatric and Environmental Dermatology, Graduate School of Medical

11 Sciences, Nagoya City University, Nagoya, Japan

12 6. Mandom Corporation, Osaka, Japan

13 7. Laboratory of Vaccine Science, WPI Immunology Frontier Research Center (IFReC),

14 Osaka University, Osaka, Japan

15 8. Division of Vaccine Science, The Institute of Medical Science, The University of Tokyo,

16 Tokyo, Japan

$17 *$ Corresponding authors

\section{Abstract}

19 Primary cilia are a unique organelle, known to provide a signaling hub for variety of cell activities. Their potential role(s) in human immune homeostasis and diseases, however, have yet to be explored. Here, we show that human dendritic cells (DCs) express primary cilia-like structure. The primary cilia growth during DC proliferation by GM-CSF was shut off by DC maturation agents, suggesting the role of primary cilia to transduce proliferation signaling. PDGFR $\alpha$ pathway, one of proliferation signal in primary cilia, promoted DC proliferation in a

25 dependent manner of intra-flagellar transport system. In epidermis with atopic dermatitis

26 patients, aberrant ciliated langerhans cells and keratinocytes with showing immature state were observed that may play a potential role in inflammation and skin barrier disorder.

Main

Primary cilia are unique organelles protruding into extracellular spaces from a basal 30 body, and work as a platform for signaling pathways [1]. Intraflagellar transport (IFT) system 31 is essential for axonome elongation and ciliary protein transport [2]. IFT gene mutations or 
32 disruption of this transport system eliminate primary cilia, resulting developmental defects, 33 signaling defects and ciliopathy [3-5]. Primary cilia are formed when cells are in G0 or G1 34 phase, and their components regulate cell cycle progression [6]. Thus, primary cilium

35 formation and the cell cycle tightly regulate each other; it is widely thought that primary cilia regulate cell proliferation and differentiation in many types of cells or tissues. While it has been well accepted that almost all types of cells can generate primary cilia, whether immune cells and/or stromal cells in immune organs/tissues express primary cilia and if so, what would be the precise roles its existence in the immune system and mechanisms of their action have not been explored until recently. In 2015, Prosser and Morrison reported that immortalized T and B cells have primary cilia [7]. Furthermore, Ezratty et al., reported that mouse embryonic epidermal keratinocytes $(\mathrm{KC})$ has primary cilia, regulating $\mathrm{KC}$ differentiation [8].

Skin is the biggest tissue, which plays immunologically important roles in its homeostasis as well as pathological conditions such as injection and injury. Amongst three layers of the skin; epidermis, dermis, and subcutaneous tissue, $\mathrm{KCs}$ are the major cells in epidermis, and its proliferation and differentiation are strictly regulated to maintain skin homeostasis [9]. KCs finally form the stratum corneum working as physical barrier against pathogens [10]. Immune cells, including Langerhans cells (LCs), which have a similar role as dendritic cells (DCs), also exist in epidermis, and maintain skin homeostasis by working as antigen-presenting cells to activate T cells [11-13]. It has long been accepted that LCs have a pivotal role in bridging innate and acquired immunity, which is required for skin homeostasis. On the other hand, they are involved in the pathology of allergic skin diseases, including atopic dermatitis [1416]. Yet, there are very few studies exploring the potential role of primary cilia in LC and/or other DCs of the skin in relation with the stromal cells such as KCs in either homeostasis or pathogenesis of skin immunity.

The prevalence of atopic dermatitis has increased greatly in the past 30 years, and it is widely known that environmental factors such as mite antigen in house dust can trigger atopic disease. In both the early and chronic stages of atopic dermatitis, type 2 immune responses, which are characterized as the elevation of several type 2 cytokines and $\operatorname{IgE}$ production, are dominant [17]. Chemokines secreted from KCs are upregulated, recruiting Th2 cells which strongly induce Th2 responses [18]. Atopic dermatitis often features skin barrier disruption,

63 leading to dryness, itchiness, and invasion by pathogens such as Staphylococcus aureus.

64 Topical steroids, tacrolimus, and moisturizers are use in its treatment, but because of its complicated pathogenesis, it often recurs after improvement. As such, it is clinically 
66

67

68

69

70

important to regulate inflammation and skin barrier maintenance, targeting both KCs and immune cells, however, potential roles of primary cilia in the pathogenesis of atopic dermatitis have not been fully clarified.

We therefore hypothesized that there may be potential role of primary cilia in the immune system in both homeostatic and pathological conditions, and examined whether human immune cells such dendritic cells express primary cilia, derived from primary blood monocytes and cell lines in vitro as well as human skin samples of healthy and atopic dermatitis patients. We further went on analyzing the molecular mechanism(s) by which expression and/or growth of primary cilia in human immune cells are regulated, and their physiological relevance in homeostasis and pathological condition of the skin.

\section{Results}

\section{Human primary immune cells have primary cilium-like structures}

To examine the primary cilia expression in human skin, we took healthy human skin samples and visualized the primary cilia in epidermis and dermis by staining with acetylated tubulin using fluorescent microscopy. As expected, we found many ciliated cells in dermis, which suggests that most ciliated cells were fibroblasts (extended data Fig. 1A). We found primary cilium-like structure in the epidermal basal area where proliferating $\mathrm{KC}$ stem cells are populous. Primary cilia-like structures were also detected in the stratum spinosum, at a lower frequency than in the basal layer (Fig. 1B). We hypothesized that most of the ciliated epidermal cells were KCs. However, terminal differentiation of KCs induces programmed cell death in the granular layer to form the stratum corneum [19]. As loss of cilia induces apoptosis in HeLa cells [20], we hypothesized that the ciliated cells in the striatum layer were not KCs but were resident living immune cells. When examining primary cilia in CD4+ T cells, CD8+ T cells, and Langerhans cells (LCs), we found only LCs in epidermis, positively merged with acetylated tubulin (Fig. 1B). Of note, most primary cilium-like structures in the stratum spinosum were not co-localized with langerin-positive cells. We did not determine the type of cells ciliated in this layer, but we hypothesized that they were KCs just before apoptosis. Mouse KCs have primary cilia ([8]), so human adult KCs may also be ciliated.

LCs are a kind of DC and have a similar function as conventional DCs (cDCs). To see whether immune cells in blood, especially cDCs, are ciliated, we isolated peripheral blood mononuclear cells (PBMCs), a mixture of immune cells, from human peripheral blood, and immunostained them with acetylated or glutamylated tubulin. Nearly $2 \%$ of cells had primary 
98 cilium-like structures a showing single protrusion stained with stabilized tubulin, extending

99 from the pericentrin by nearly $1 \mu \mathrm{m}$ (Fig. 1C). We examined whether the structure was

100 Arl13B positive. Arl13B is known as primary cilia marker, and is required for cilia formation

101 and maintenance [21]. Arl13B-GFP was exogenously expressed in PBMCs, then cells were

102 immunostained with green fluorescent protein (GFP). A GFP signal was observed as a single

103 linear structure (Fig. 1D) similar to the stabilized tubulin (Fig. 1C). As the formation of

104 primary cilia is strongly associated with the cell cycle and serum starvation promotes their

105 elongation in many types of cells by inducing cell cycle arrest [22, 23], we examined whether

106 human primary immune cells use a similar mechanism to elongate primary cilia. Serum

107 starvation by culturing in $0.5 \%$ serum for $16 \mathrm{~h}$ significantly increased the frequency of

108 primary cilium-like structures, which tended to be longer than those in cells treated in $10 \%$

109 serum (Fig. 1E, F). For further investigation of primary cilium-like structures in PBMCs in

110 detail, we used transmission electron microscopy. We observed the vesicle-centrosome

111 interaction, which resembles a ciliary vesicle (Fig. 1G). Also, centrosome elongation

112 resembling axoneme extension, which is found in early primary cilium elongation, was

113 clearly observed (Fig. 1G). These results raise the strong possibility that human immune cells

114 are ciliated, and have similar machinery to elongate primary cilia.

115 To identify specific types of ciliated immune cells, we isolated monocytes, cDCs,

116 plasmacytoid dendritic cells (pDCs), CD4 T cells, CD8 T cells, natural killer cells, and B cells

117 from human PBMCs by flow cytometry and immunostained them with acetylated tubulin.

118 Except for CD8 T cells, all other cells had primary cilium-like structures resembling that in

119 Figure 1C (Fig. 1G, Supp. Table 1). cDCs had the highest rate, so we focused on the function

120 of primary cilia in DCs. The frequency of ciliated primary DCs isolated from PBMCs was

121 comparable to that of LCs in epidermis (Fig. 4D, Supp. Table 1). In summary, these results

122 suggest the presence of primary cilia in DCs and LCs. We next sought the function of primary

123 cilia in these cells.

\section{DC maturation decreases primary cilia}

125 DCs can be differentiated from monocytes in vitro [24]. To analyze whether monocyte-

126 derived DCs are ciliated in vitro, we isolated CD14+ monocytes from human PBMCs and

127 cultured them for 7 days with stimulation with GM-CSF, IL-4 and TNF $\alpha$ to differentiate into

128 mature DCs. When we looked at primary cilia in them, the frequency of primary cilia was

129 significantly decreased as day passed (Fig. 2A). To analyze whether DC maturation decreases

130 primary cilium formation, we stimulated primary DCs with TNF $\alpha$ or PGE2 for $24 \mathrm{~h}$. TNF $\alpha$ 
131 significantly decreased primary cilium formation dose-dependently (Fig. 2B). PGE2

132 stimulation had a similar effect (Fig. 2C), which suggests that DC maturation decreased 133 primary cilium formation.

134 We also induced immature DCs by culturing with GM-CSF and IL-4. In immature DCs 135 at day 7 , nearly $1 \%$ of cells were ciliated, similar to the proportion in primary DCs (Fig. 2D, 136 Supp. Table 1). The percentage did not differ significantly from day 1 to day 7 (Fig. 2D).

137 Most DCs in healthy blood are immature, so these results suggest that immature DCs derived 138 from CD14+ monocytes in vitro are similar to primary DCs, and raise the possibility that 139 primary cilium formation was inhibited while monocytes differentiated into mature DCs. 140 Next, we investigated whether IL-4 or GM-CSF promoted primary cilium formation, 141 because the frequency of primary cilia in immature DCs was a little higher than that in 142 untreated (day 0) monocytes (Fig. 2D). GM-CSF significantly promoted primary cilium 143 formation (Fig. 2E). Interestingly, IL-4 did not increase it, whereas IL-4 was simultaneously 144 added with GM-CSF. It is widely known that cell maturation and cell proliferation show 145 inverse behaviors. Immature or precursor cells proliferate much more than mature cells. To 146 investigate the effect of GM-CSF and IL-4 on cell proliferation, we cultured immature DCs

147 with GM-CSF or IL-4 and analyzed cell growth by MTT assay. GM-CSF increased DC 148 proliferation, but IL-4 did not. Co-treatment with IL-4 canceled the effect of GM-CSF (Fig.

149 2F). These findings raise an interesting possibility that primary cilium formation is correlated 150 with DC proliferation, making GM-CSF a candidate to promote primary cilium formation.

151 Having demonstrated that GM-CSF promoted primary cilium formation and cell

152 proliferation, we evaluated which cells secrete GM-CSF. We stimulated CD14+ monocytes to 153 differentiate into immature and mature LCs, then compared GM-CSF expression between 154 those and human immortalized KCs (HaCaTs). Immature LCs expressed GM-CSF much 155 more than mature LCs and HaCaTs did (Fig. 2G). Immature LCs spontaneously expressed 156 GM-CSF without any stimulation, and stimulation did not alter its expression (Fig. 2G). IL-4 157 was not detected in LCs, but HaCaTs spontaneously expressed it (extended data Fig. 2).

158 These results suggest that immature LCs in epidermis are the main producers of GM-CSF.

159 Df promotes primary cilium formation.

160 As GM-CSF promoted ciliogenesis and proliferation, we next focused on 161 immunostimulants that promote GM-CSF secretion. GM-CSF is a Th2 cytokine, and its 162 expression is elevated in atopic dermatitis [25], which can be triggered by house dust mite 163 antigen. Therefore, we tested the effect of mite antigen on ciliogenesis. We also tested LPS, 
164 which activates TLR4 and induces strong Th1 immune responses in human immature DCs.

165 We stimulated primary DCs with LPS, and mite antigen derived from Dermatophagoides

166 farinae ( $D f)$. $D f$ antigen slightly (but not significantly) increased the population of ciliated

167 DCs (Fig. 3A). In contrast, LPS significantly decreased primary cilia (extended data Fig. 4A).

168 We next analyzed how these immunostimulants stimulate cell proliferation activity. Contrary

169 to expectation, LPS and $D f$ treatment did not affect proliferation activity in DCs (Fig. 3B,

170 extended data Fig. 3B).

171 KCs showed different responses to immunostimulants from DCs. LPS stimulation

172 decreased KC proliferation while $D f$ significantly increased it (extended data Fig. 3C). Ki67

173 expression in HaCaTs was increased as expected after stimulation with $D f$ (extended data Fig.

174 3D). The differences in cell responses between primary DCs and HaCaTs are not surprising,

175 but we hypothesized that Df is one of the important molecule to regulate DCs and LCs

176 functions.

177 PDGFRa signaling promotes DC proliferation

178 PDGFR $\alpha$ is highly localized in primary cilia [26]. PDGF-A, a specific ligand for PDGFR $\alpha$,

179 promotes fibroblast and $\mathrm{KC}$ proliferation during wound healing of skin [9]. We identified

180 PDGFR $\alpha$ expression in primary DCs and HaCaTs (extended data Figs. 3D, 4B, 4D). To

181 identify the type of cells secreting PDGF-A, we performed ELISA assay with culture

182 supernatant of immature LCs, mature LCs, and HaCaTs. HaCaTs spontaneously expressed

183 PDGF-A, but LCs did not. These results strongly suggest that KCs have a major role in 184 secreting PDGF-A (extended data Fig. 4A).

185 Next we analyzed the function of PDGFR $\alpha$ signaling on proliferation in primary DCs and

186 KCs. Co-treatment of DCs or KCs with PDGF-AA and GM-CSF significantly increased

187 proliferation (Fig. 3C, extended data Fig. 4C, 4D). PDGFR $\alpha$ expression was not changed

188 when cells were stimulated with PDGF-AA, GM-CSF, LPS and Df (extended data Fig. 3D,

189 4B, 4D). To investigate whether disruption of primary cilia causes DC maturation, we

190 performed a knockdown experiment using siRNA targeting IFT88. Knockdown of IFT88

191 increased the CD86 and CCR7 expression in THP1-derived immature DCs (Fig. 3D).

192 Furthermore, ki67 expression was downregulated with IFT88 knockdown (Fig. 3E).

193 Interestingly, knockdown of IFT88 canceled proliferation promoted by co-stimulation with

194 PDGF-AA and GM-CSF (Fig. 3F). These results suggest an important role of the PDGFR $\alpha$

195 signaling pathway through primary cilia in DC proliferation.

196 Primary cilia are increased in epidermis with atopic dermatitis 
LCs proliferate extensively in epidermis with atopic dermatitis ([27], Fig. 4A). To address whether atopic condition alters primary cilium formation, we investigated the frequency of primary cilia in human epidermis with atopic dermatitis. Compared with healthy skin, the number of primary cilia was greatly increased especially in the basal area (Fig. 4B, D). Interestingly, ciliated cells other than LCs were often detected in atopic dermatitis (Fig. 4B,C). The frequency of ciliated cells (all types) and of ciliated LCs in atopic LCs was increased (Fig. 4D). We did not determine ciliated cell type other than LC, but we speculated they were $\mathrm{KC}$ because majority of epidermal cells are $\mathrm{KC}$.

We also investigated the number of proliferating cells in atopic epidermis. Ki67 is a proliferation marker, and its expression is highly increased in $\mathrm{S}$ and $\mathrm{M}$ phases. In atopic dermatitis, ki67 positive LC is increased [27], and we found similar results. Ki67-positive epidermal cells were significantly increased in atopic epidermis (Fig. 4E). Unexpectedly, some ciliated cells were positively stained for ki67 in atopic epidermis, but none were positive in healthy skin samples (Fig. 4F). Primary cilia are generally formed in G0 or G1

211 phase, and their formation is repressed in proliferating cells in G2/M phase ([6]). We did not

212 determine the specific cell cycle phase that cells were in, but strong expression of ki67 in

213 atopic epidermis suggests that ki67 positive atopic epidermal cells were in S or G2/M phase.

214 It is widely accepted that proliferation and maturation are highly correlated, so we next

215 investigated an LC maturation marker, CCR7, in epidermis. In healthy epidermis, nearly 35\%

216 of LCs were positively stained with CCR7, but atopic LCs were not (Fig. 4G). This result 217 strongly indicates that atopic LCs are immature.

218 We also explored the expression of KC maturation markers. In healthy epidermis, K14, a 219 marker of immature KCs, was highly expressed near the basal layer, and K10, a marker of 220 mature KCs, was highly expressed only in the stratum granulosum (Fig. 5A). In atopic epidermis, in contrast, K14 was highly expressed in the basal layer and moderately expressed throughout the stratum spinosum and stratum granulosum (Fig. 5B). Interestingly, K10 was strongly expressed even in the stratum spinosum, along with K14 (Fig. 5B). We next investigated other markers of differentiation, loricrin and filaggrin. These proteins are necessary for skin barrier formation, and both are decreased in more than $20 \%$ of patients. It is widely accepted that barrier disruption increases the risk of atopic dermatitis [28-30]. The percentage of ciliated cells was significantly increased in patients with low levels of loricrin, but was not correlated with filaggrin expression (Fig. 5C, extended data Fig. 5). Interestingly, examining the correlation between $\operatorname{IgE}$ level and Loricrin expression, as well $\operatorname{IgE}$ level and

230 primary cilia did not have significant differences (Fig. 5D, E). These results suggest the 
231 physiological importance of primary cilia in KCs to maintain adequate loricrin expression and

232 skin barrier formation, which develops but not exacerbate atopic dermatitis. In summary,

233 immunostaining results strongly suggested excessive proliferation and abnormal primary

234 cilium formation in atopic dermatitis, which in turn caused hyperproliferation and sustenance

235 of immature LCs and KCs in atopic dermatitis.

236 Immature Langerhans cells secrete chemokines

237 Our findings show that ciliated DCs and LCs were immature and highly proliferative in

238 vitro and in atopic dermatitis. We next sought the physiological function of immature LCs.

239 Th2 T cells highly infiltrate atopic epidermis and secrete Th2 cytokines [18]. Thus, we asked

240 whether immature LCs secrete chemokines to recruit Th2 T cells. To answer this, we

241 analyzed chemokine expression. MCP1 was highly secreted by immature LCs relative to

242 mature LCs and KCs (extended data Fig. 6A). In contrast, TARC/CCR17 was expressed by

243 mature LCs, but not so much by immature LCs or KCs (extended data Fig. 6B). MDC was

244 highly expressed in both immature and mature LCs but was not detected in KCs (extended

245 data Fig. 6C). These data suggest the important role of LCs but not KCs in secreting

246 chemokines to recruit Th2 cells. In atopic dermatitis, immature LCs were highly increased

247 (Fig. 4G). Our results raise the strong possibility that immature LCs are the main releasers of

248 Th2 chemokines in atopic dermatitis, which exacerbate disease.

\section{Discussion}

250 We identified primary cilia in human epidermis and immune cells from blood (Fig. 1).

251 Primary cilium formation in epidermis was highly promoted in atopic dermatitis (Fig. 4).

252 High correlation of primary cilium formation and proliferation suggests a primary function of

253 cilia in epidermal cell proliferation in atopic dermatitis. In atopic epidermis, ki67-positive

254 ciliated cells were greatly increased. Primary cilium formation is basically inhibited in G2/M

255 and $\mathrm{S}$ phase, and this phenotype was not found in healthy epidermis, so the cell cycle and

256 proliferation may be disrupted in atopic dermatitis which maybe a cause of disease. Our data

257 suggested that GM-CSF promotes primary cilium formation, and PDGFR $\alpha$ signaling via

258 primary cilia promotes proliferation of DCs (Figs. 2-4). The candidate of atopic dermatitis

259 inducer, Df, induced primary cilia formation in DCs and proliferation in KCs. Because KCs

260 produce PDGF-AA constantly, Df may induce thickening of epidermis, which strongly

261 induces LC proliferation. We have not identified what induces atopic dermatitis, however, we

262 propose that GM-CSF and Df are strong candidates to develop atopic dermatitis by regulating 
263 primary cilia formation and proliferation. Interestingly, ciliopathy patients in several

264 pedigrees show frequent atopic dermatitis or asthma along with other phenotypes caused by

265 primary cilium defect $[31,32]$. We have not identified genetic alteration of cilium-related

266

267

268

269

270

271

272

273

274

275

276

277

278

279

280

281

282

283

284

285

286

287

288

289

290

291

292

293

294

295

296

genes in atopic dermatitis patients, so further experiments will be required.

Interestingly, the purchased HaCaTs and primary KCs were not ciliated in vitro, although epidermal KCs in tissue were (data not shown; Figs. 1, 4). Because of this technical difficulty, we could not study the function of primary cilia in KCs in detail. The differences between epidermal KCs and isolated KCs are unknown, but 3D cell-cell interaction or environmental factors in tissue may be involved in primary cilium formation. Further investigation is required to explain the function of primary cilia in KCs.

Proliferation and maturation are highly inversely correlated with each other. Therefore, we asked whether inhibition of proliferation by disrupting primary cilia promoted maturation. We demonstrated that knockdown of IFT88 in THP1-derived DCs promoted maturation by attenuating proliferation activity promoted by PDGFR $\alpha$ signaling (Fig. 3D, E). These results raise a strong possibility that PDGFR $\alpha$ signaling in primary cilia regulates cell proliferation and inhibits maturation. We tried to identify the expression of PDGFR $\alpha$ in both healthy and atopic epidermis, but the experiment did not work (data not shown). Furthermore, we could not detect the PDGFR $\alpha$ localization in primary cilia in LCs and DCs, even though these cells expressed PDGFR $\alpha$ detected by western blotting (extended data Figs. 4, 5). Interestingly, we found constitutive expression of PDGF-AA in HaCaTs (extended data Fig. 5A). This result raises a possibility that PDGF-AA expression is higher in atopic skin because the number of KCs is obviously increased. The relationship between pathophysiology and PDGF-AA expression will be the next topic of research. Imatinib, a PDGFR $\alpha$ antagonist, treats asthma by decreasing mast cell activation in patients and by decreasing MCP1 expression in mice $[33,34]$. Its effectiveness in the treatment of atopic dermatitis has not been elucidated, but these reports suggest the importance of PDGFR $\alpha$ signaling in allergic disorders. Furthermore, methotrexate, a folate antagonist, successfully treated atopic dermatitis [35, 36]. Previously we demonstrated that folate metabolic pathway is required for primary cilium formation [37]. These knowledge support our idea that the regulation of primary cilia or PDGFR $\alpha$ signaling could help in the treatment of atopic dermatitis.

On the basis of our novel findings, we propose that primary cilia in LCs or in KCs have an important role in skin homeostasis by regulating proliferation, and excess cilium formation may cause atopic dermatitis. Over-formation of primary cilia in KCs sustains immaturity, impairing barrier function by reducing loricrin expression. Ciliated immature LCs strongly 
297 recognize antigens or immunostimulants that pass through the skin barrier, and LCs secrete

298 GM-CSF. GM-CSF promotes primary cilium formation in both KCs and LCs, and

299 proliferation signals, including PDGFR $\alpha$, are transduced more strongly. Chemokines secreted

300 from immature LCs strongly recruit Th2 T cells, which causes a vicious cycle of atopic

301 dermatitis (extended data Fig. 6D).

\section{Methods}

\section{Human skin samples}

\section{Isolation of primary DCs and CD14+ monocytes}

305 Samples of human whole peripheral blood were purchased from the Japanese Red Cross

306 Society according to the Guidelines on the Use of Donated Blood in R\&D, etc. Blood from 89

307 healthy donors was drawn into a heparinized syringe and diluted with an equal volume of

308 phosphate-buffered saline (PBS). A 35-mL volume of diluted blood was layered over $15 \mathrm{~mL}$

309 of Ficoll-Paque Plus density gradient medium (GE Healthcare) in Leucosep 50-mL tubes

310 (Greiner Bio-One), then centrifuged at $500 \times \mathrm{g}$ for $20 \mathrm{~min}$ at room temperature without

311 braking. The PBMC fraction was carefully collected by pipetting, and the cells were washed

312 with RPMI1640 supplemented with $10 \%$ fetal bovine serum (FBS). After centrifugation at

$313500 \times g$ for $10 \mathrm{~min}$ at room temperature, red blood cells were lysed with Ammonium-

314 Chloride-Potassium (ACK) buffer (150 mM NH $4 \mathrm{Cl} 10 \mathrm{mM}$ KHCO3, $0.1 \mathrm{mM}$ EDTA in PBS)

315 in a conical tube at room temperature for $10 \mathrm{~min}$. After washing with RPMI1640 containing

316 10\% FBS, CD14-positive monocytes were isolated by CD14 microbeads, human (130-050-

317 201, Miltenyi Biotec). The positive fraction was used as CD14-positive monocytes. DCs were

318 isolated with a Blood Dendritic Cell Isolation Kit II, human (130-091-379, Miltenyi Biotec),

319 from the negative fraction of the CD14-positive fraction.

\section{Cell culture}

321 HaCaT cells were cultured in Dulbecco's modified Eagle's medium (DMEM) supplemented

322 with $10 \%$ FBS, $1 \%$ penicillin and $1 \%$ streptomycin. Immature and mature LCs were induced

323 to differentiate from CD14-positive monocytes derived from human primary PBMCs. To

324 induce immature LCs, CD14-positive monocytes were cultured in RPMI1640 supplemented

325 with $10 \mathrm{ng} / \mathrm{mL}$ IL-4 (100-09, Shenandoah), $100 \mathrm{ng} / \mathrm{mL}$ GM-CSF (100-08, Shenandoah), and

$32610 \mathrm{ng} / \mathrm{mL}$ TGF $\beta$ (240-B, R\&D Systems). To induce mature LCs, CD14-positive monocytes

327 were cultured in RPMI1640 supplemented with 10 ng/mL IL-4, 100 ng/mL GM-CSF, 10

$328 \mathrm{ng} / \mathrm{mL}$ TGF $\beta$, and $20 \mathrm{ng} / \mathrm{mL}$ TNF $\alpha$ (MAN0003622, Gibco). A half volume of fresh medium 
was added every 2 days.

330 Isolation of monocytes, $p D C s$, and cDCs by flow cytometry

331 Human PBMCs were labeled with Alexa 647 anti-human CD11c (301620, clone 3.9,

332 BioLegend), anti-human HLA-DR (Class III) PE-Texas conjugate (MHLDR17, Life

333 Technology), BV421 anti-human CD14 (325627, clone HCD14, BioLegend), PE-Cy7 anti-

334 human CD123 (560826, clone 7G3, BD Biosciences), APC-Fire 750 anti-human CD8a

335 (301065, clone RPA-T8, BioLegend), APC-Fire 750 anti-human CD20 (302357, clone 2H7,

336 BioLegend), and APC-H7 anti-human CD3 (560275, clone SK7, BD Pharmingen) in a

337 Live/Dead Fixable Aqua Dead Cell Stain Kit (L34966, Invitrogen). Labeled cells were

338 analyzed and isolated using BD FACSAria II (BD Biosciences). HLA-DR ${ }^{\text {high }}-\mathrm{CD} 14^{\text {low }}$ cells

339 were gated from a CD3-, CD8-, CD20-negative live-cell population and identified as the DC

340 population. CD123 ${ }^{\text {middle }}-\mathrm{CD} 11 \mathrm{c}^{\text {high }}$ cells in the DC population were determined as cDCs.

341 CD123 $3^{\text {high }}-$ CD $11 c^{\text {low }}$ cells in the DC population were determined as pDCs. CD14 ${ }^{\text {high }}-$ HLA-

342 DR ${ }^{\text {high }}-\mathrm{CD} 11 \mathrm{c}^{\text {high }}-\mathrm{CD} 123^{\text {middle }}$ cells were determined as monocytes.

343 ELISA

344 In 48-well plates, $2.0 \times 10^{5} \mathrm{HaCaT}$ cells were stimulated with $100 \mu \mathrm{L}$ of DMEM

345 supplemented with $0.5 \%$ FBS. In 96-well plates, $2.0 \times 10^{4}$ LCs were stimulated with $100 \mathrm{~mL}$

346 of RPMI1640 supplemented with $0.5 \%$ FBS. After 24 h stimulation, 0.5\% Triton X-100 was

347 added into the cell cultures and then cell lysate was collected with supernatant. Cell lysate

348 was used for ELISA assay following the kit protocol. ELISA kits (CCL17, DY364, MCP1,

349 DCP00, MDC, DMD00, IL-4, D4050, GM-CSF, DY215) were purchased from R\&D

350 Systems. PDGF-AA ELISA kits (EHPDGF) were purchased from Thermo Fisher Scientific.

351 Absorbance (excitation, $450 \mathrm{~nm}$ ) was measured on a microplate reader (Infinite F200 Pro,

352 Tecan). As reference, background absorbance was measured at $560 \mathrm{~nm}$.

\section{Immunostaining}

354 Paraffin-embedded human skin tissue was deparaffinized in xylene 2 times for 10 min each.

355 The samples were then immersed in 100\% ethanol, 95\% ethanol, and 2 lots of deionized

356 water for $10 \mathrm{~min}$ each. After rehydration, antigens were retrieved: The samples were

357 immersed in $1 \mathrm{mM}$ EDTA in deionized water and boiled by microwave for $15 \mathrm{~min}$. They

358 were then incubated with blocking buffer (10\% FBS, $0.1 \%$ Triton X-100 in PBS) at room

359 temperature for $1 \mathrm{~h}$. First antibodies (listed below) were diluted 1:1000 in PBS for the

360 detection of acetylated tubulin, langerin, and ki67. CCR7 antibody was diluted 1:100 and

361 incubated at $4{ }^{\circ} \mathrm{C}$ overnight. After 3 washes in wash buffer $(0.1 \%$ Tween-20 in PBS), second 
362 antibodies (Alexa 488-conjugated IgG, Alexa 594-conjugated IgG) containing 1/5000

363 Hoechst 33342 (Thermo Fisher Scientific) were reacted at room temperature for $2 \mathrm{~h}$. After

364 rinsing in wash buffer, samples were mounted with ProLong Gold antifade reagent (Thermo

365 Fisher Scientific). For single-cell immunostaining following the blocking step, cells were

366 mounted on MAS-coated slide glass (Matsunami). First antibodies comprised anti-acetylated

$367 \alpha$-tubulin (T7451, clone 6-11B-1, Sigma), anti-langerin (ab192027, clone EPR15863,

368 Abcam), anti-ki67 (\#9449, clone 8D5, Cell Signaling Technology; ab16667, clone SP6,

369 Abcam), anti-CCR7 (MAB197, clone 150503, R\&D Systems), anti-pericentrin (A301-348A,

370 Bethyl), and anti-GFP (SC-9996, clone B-2, Santa Cruz Biotechnology) antibodies.

371 The number of ciliated cells was counted and statistically analyzed in Prism 7 software.

372 Proliferation assay

373 In 96-well plates, $3000 \mathrm{HaCaT}$ cells or 5000 primary DCs were stimulated with reagent in

$374100 \mu \mathrm{L}$ of medium containing $0.5 \%$ FBS and $10 \mu \mathrm{L}$ of CCK-8 buffer (Cell Counting Kit-8,

375 Dojindo). After $24 \mathrm{~h}$, absorbance at $450 \mathrm{~nm}$ was measured on a microplate reader (Infinite

376 F200 Pro). As reference, background absorbance was measured at $560 \mathrm{~nm}$.

377 Electron microscopy

378 Cells were fixed with $2 \%$ glutaraldehyde in $0.1 \mathrm{M}$ phosphate buffer $(\mathrm{PB}), \mathrm{pH} 7.4$, at $4{ }^{\circ} \mathrm{C}$

379 overnight. The fixed samples were washed 3 times with $0.1 \mathrm{M}$ PB for $30 \mathrm{~min}$ each, and were

380 post-fixed with $2 \% \mathrm{OsO}_{4}$ in $0.1 \mathrm{M} \mathrm{PB}$ at $4{ }^{\circ} \mathrm{C}$ for $1 \mathrm{~h}$. They were then dehydrated in a graded

381 ethanol solution at $50 \%$ and $70 \%$ for $5 \mathrm{~min}$ each at $4{ }^{\circ} \mathrm{C}, 90 \%$ for $5 \mathrm{~min}$ at room temperature,

382 and 3 changes of $100 \%$ for 5 min each at room temperature. The samples were infiltrated with

383 propylene oxide (PO) 2 times for 5 min each, put into a 70:30 mixture of PO and resin

384 (Quetol-812; Nisshin EM Co.) for $10 \mathrm{~min}$, and then exposed to the open air overnight for the

385 PO to volatilize. The samples were transferred to fresh $100 \%$ resin and polymerized at $60{ }^{\circ} \mathrm{C}$

386 for $48 \mathrm{~h}$. The polymerized resins were ultrathin-sectioned at $70 \mathrm{~nm}$ with a diamond knife on

387 an ultramicrotome (Ultracut UCT; Leica), and the sections were mounted on copper grids.

388 They were stained with $2 \%$ uranyl acetate at room temperature for $15 \mathrm{~min}$, washed with

389 distilled water, and then secondary-stained with lead stain solution (Sigma-Aldrich) at room

390 temperature for $3 \mathrm{~min}$. The grids were observed by transmission electron microscope (JEM-

3911400 Plus; JEOL Ltd.) at an acceleration voltage of $100 \mathrm{kV}$. Digital images $(3296 \times 2472$

392 pixels) were taken with a CCD camera (EM-14830RUBY2; JEOL Ltd.).

393 Reference mendeley

394 


\section{References}

396 1. Goetz, S.C. and K.V. Anderson, The primary cilium: a signalling centre during vertebrate development. Nat Rev Genet, 2010. 11(5): p. 331-44.

2. Pedersen, L.B. and J.L. Rosenbaum, Intraflagellar transport (IFT) role in ciliary assembly, resorption and signalling. Curr Top Dev Biol, 2008. 85: p. 23-61.

3. Fliegauf, M., T. Benzing, and H. Omran, When cilia go bad: cilia defects and ciliopathies. Nat Rev Mol Cell Biol, 2007. 8(11): p. 880-93.

4. Valente, E.M., et al., Primary cilia in neurodevelopmental disorders. Nat Rev Neurol, 2014. 10(1): p. 27-36.

5. Nigg, E.A. and J.W. Raff, Centrioles, centrosomes, and cilia in health and disease. Cell, 2009. 139(4): p. 663-78.

6. Goto, H., A. Inoko, and M. Inagaki, Cell cycle progression by the repression of primary cilia formation in proliferating cells. Cell Mol Life Sci, 2013. 70(20): p. 3893-905.

7. Prosser, S.L. and C.G. Morrison, Centrin2 regulates CP110 removal in primary cilium formation. J Cell Biol, 2015. 208(6): p. 693-701.

8. Ezratty, E.J., et al., A role for the primary cilium in Notch signaling and epidermal differentiation during skin development. Cell, 2011. 145(7): p. 1129-41.

9. Barrientos, S., et al., Growth factors and cytokines in wound healing. Wound Repair Regen, 2008. 16(5): p. 585-601.

10. Madison, K.C., Barrier function of the skin: "la raison d'etre" of the epidermis. J Invest Dermatol, 2003. 121(2): p. 231-41.

11. Pasparakis, M., I. Haase, and F.O. Nestle, Mechanisms regulating skin immunity and inflammation. Nat Rev Immunol, 2014. 14(5): p. 289-301.

12. Nestle, F.O., et al., Skin immune sentinels in health and disease. Nat Rev Immunol, 2009. 9(10): p. 679-91.

13. Deckers, J., H. Hammad, and E. Hoste, Langerhans Cells: Sensing the Environment in Health and Disease. Front Immunol, 2018. 9: p. 93.

14. Callard, R.E. and J.I. Harper, The skin barrier, atopic dermatitis and allergy: a role for Langerhans cells? Trends Immunol, 2007. 28(7): p. 294-8.

15. Dubrac, S., M. Schmuth, and S. Ebner, Atopic dermatitis: the role of Langerhans cells in disease pathogenesis. Immunol Cell Biol, 2010. 88(4): p. 400-9.

16. Nakajima, S., et al., Langerhans cells are critical in epicutaneous sensitization with protein antigen via thymic stromal lymphopoietin receptor signaling. J Allergy Clin Immunol, 2012. 129(4): p. 1048-55 e6.

17. Gandhi, N.A., et al., Targeting key proximal drivers of type 2 inflammation in disease. Nat Rev Drug Discov, 2016. 15(1): p. 35-50.

18. Nedoszytko, B., et al., Chemokines and cytokines network in the pathogenesis of the inflammatory skin diseases: atopic dermatitis, psoriasis and skin mastocytosis. Postepy Dermatol Alergol, 2014. 31(2): p. 84-91.

19. Eckhart, L., et al., Cell death by cornification. Biochim Biophys Acta, 2013. 1833(12): p. 3471-3480.

20. Kim, J.C., et al., The Bardet-Biedl protein BBS4 targets cargo to the pericentriolar region and is required for microtubule anchoring and cell cycle progression. Nat Genet, 2004. 36(5): p. 462-70.

21. Larkins, C.E., et al., Arll3b regulates ciliogenesis and the dynamic localization of Shh signaling proteins. Mol Biol Cell, 2011. 22(23): p. 4694-703.

22. Tang, Z., et al., Autophagy promotes primary ciliogenesis by removing OFD1 from centriolar satellites. Nature, 2013. 502(7470): p. 254-7. 
444 23. Orhon, I., et al., Autophagy and regulation of cilia function and assembly. Cell Death

445

446

447

448

449

450

451

452

453

454

455

456

457

458

459

460

461

462

463

464

465

466

467

468

469

470

471

472

473

474

475

476

477

478

479

480

481

482

483

484

485

486

487

Differ, 2015. 22(3): p. 389-97.

24. Nair, S., G.E. Archer, and T.F. Tedder, Isolation and generation of human dendritic cells. Curr Protoc Immunol, 2012. Chapter 7: p. Unit7 32.

25. Pastore, S., et al., Granulocyte macrophage colony-stimulating factor is overproduced by keratinocytes in atopic dermatitis. Implications for sustained dendritic cell activation in the skin. J Clin Invest, 1997. 99(12): p. 3009-17.

26. Schneider, L., et al., PDGFRalphaalpha signaling is regulated through the primary cilium in fibroblasts. Curr Biol, 2005. 15(20): p. 1861-6.

27. Chorro, L., et al., Langerhans cell (LC) proliferation mediates neonatal development, homeostasis, and inflammation-associated expansion of the epidermal LC network. $\mathrm{J}$ Exp Med, 2009. 206(13): p. 3089-100.

28. Palmer, C.N., et al., Common loss-of-function variants of the epidermal barrier protein filaggrin are a major predisposing factor for atopic dermatitis. Nat Genet, 2006. 38(4): p. 441-6.

29. O'Regan, G.M., et al., Filaggrin in atopic dermatitis. J Allergy Clin Immunol, 2008. 122(4): p. 689-693.

30. Kim, B.E., et al., Loricrin and involucrin expression is down-regulated by Th2 cytokines through STAT-6. Clin Immunol, 2008. 126(3): p. 332-7.

31. Aldahmesh, M.A., et al., IFT27, encoding a small GTPase component of IFT particles, is mutated in a consanguineous family with Bardet-Biedl syndrome. Hum Mol Genet, 2014. 23(12): p. 3307-15.

32. Moore, S.J., et al., Clinical and genetic epidemiology of Bardet-Biedl syndrome in Newfoundland: a 22-year prospective, population-based, cohort study. Am J Med Genet A, 2005. 132A(4): p. 352-60.

33. Cahill, K.N., et al., KIT Inhibition by Imatinib in Patients with Severe Refractory Asthma. N Engl J Med, 2017. 376(20): p. 1911-1920.

34. Berlin, A.A. and N.W. Lukacs, Treatment of cockroach allergen asthma model with imatinib attenuates airway responses. Am J Respir Crit Care Med, 2005. 171(1): p. 35-9.

35. Shaffrali, F.C., et al., Experience with low-dose methotrexate for the treatment of eczema in the elderly. J Am Acad Dermatol, 2003. 48(3): p. 417-9.

36. Zoller, L., M. Ramon, and R. Bergman, Low dose methotrexate therapy is effective in late-onset atopic dermatitis and idiopathic eczema. Isr Med Assoc J, 2008. 10(6): p. 413-4.

37. Toriyama, M., et al., Folate-dependent methylation of septins governs ciliogenesis during neural tube closure. FASEB J, 2017. 31(8): p. 3622-3635.

\section{Acknowledgments}

The authors thank the Japanese Red Cross Society for supplying blood samples under the Guidelines on the Use of Donated Blood in $R \& D$, etc. This work was supported in part by JSPS KAKENHI grant JP 19K17797 (to M.T.). The authors declare no conflicts of interest.

\section{Author contributions}

M.T. and K.J.I. conceived and designed the experiments and wrote the manuscript; M.T., 
bioRxiv preprint doi: https://doi.org/10.1101/2020.02.04.933333; this version posted February 20, 2020. The copyright holder for this preprint (which was not certified by peer review) is the author/funder. All rights reserved. No reuse allowed without permission.

488 D.R., and M.N. conducted the experiments; and F.F., F.O., and A. Morita contributed to the 489 writing and editing of the manuscript. 
Figure 1. Human primary immune cells have primary cilia-like structure

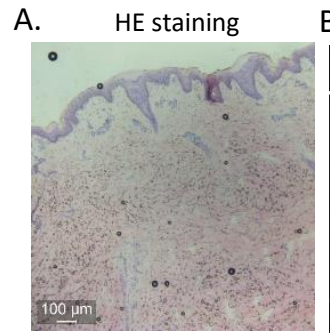

B. Acetylated tubulin
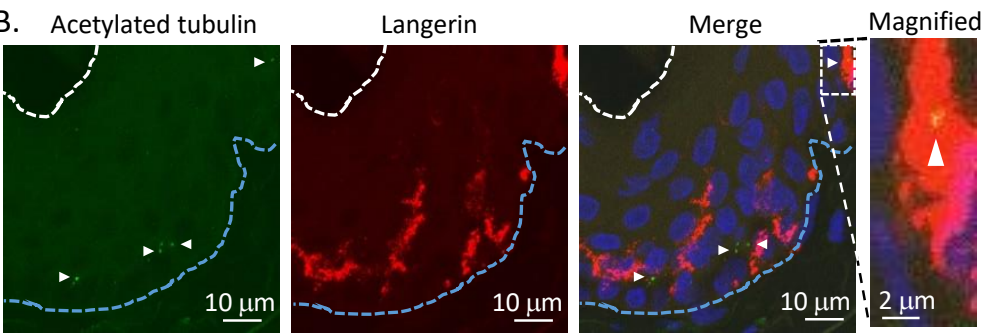

C. Acetylated tubulin
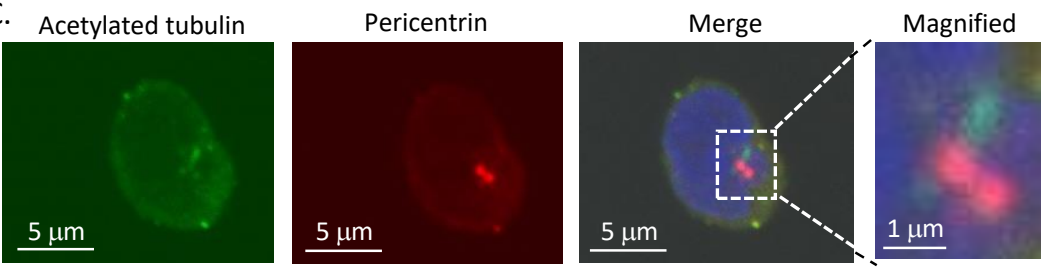

Glutamylated tubulin
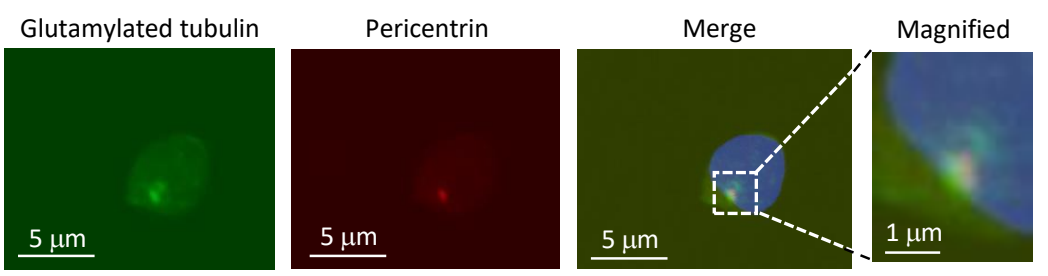

F.

G.
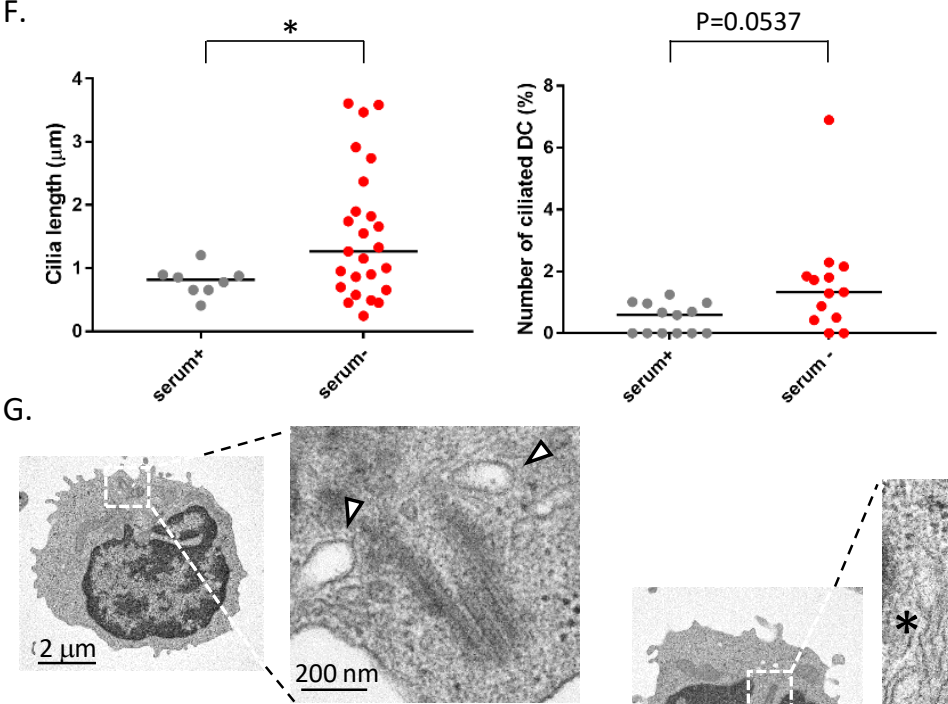

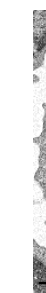
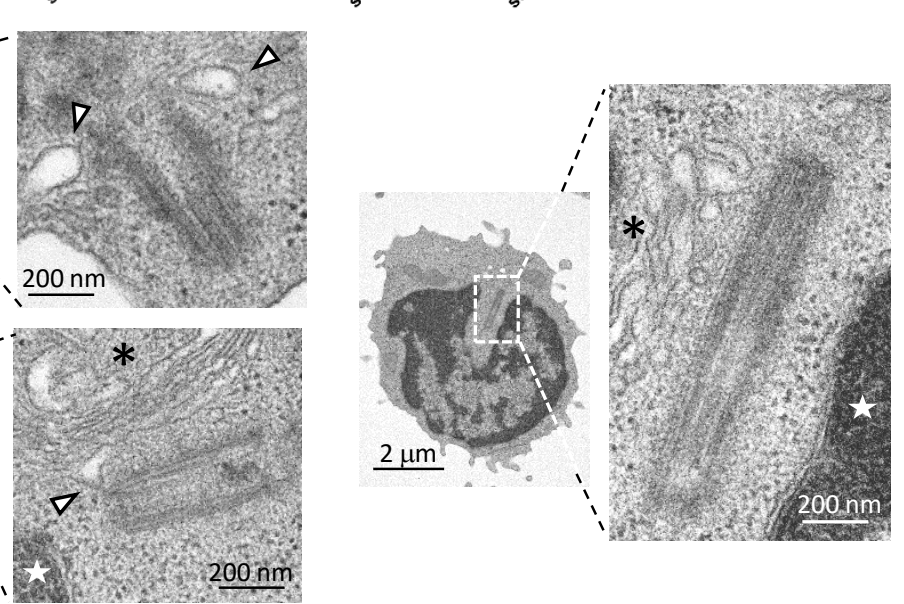

H.

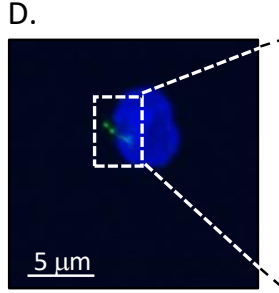

E.
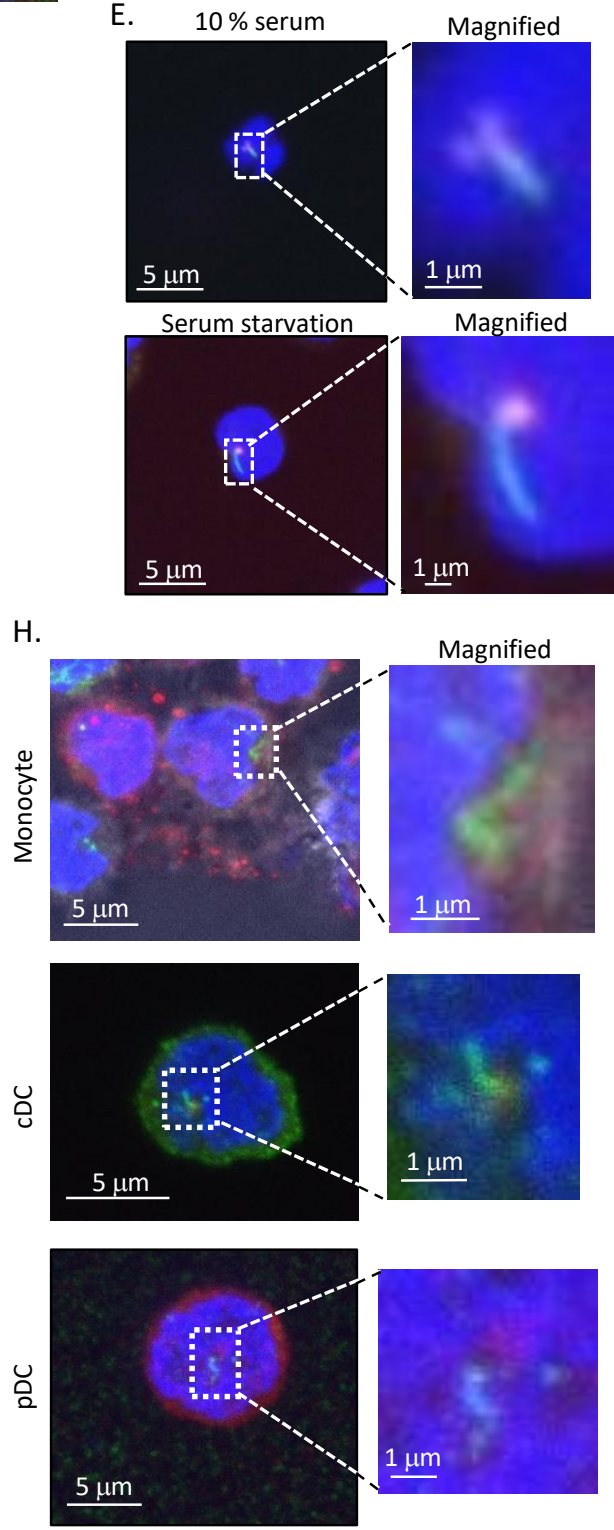

Magnified

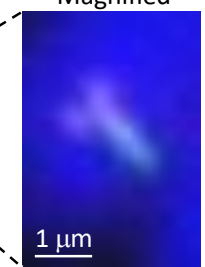

Magnified
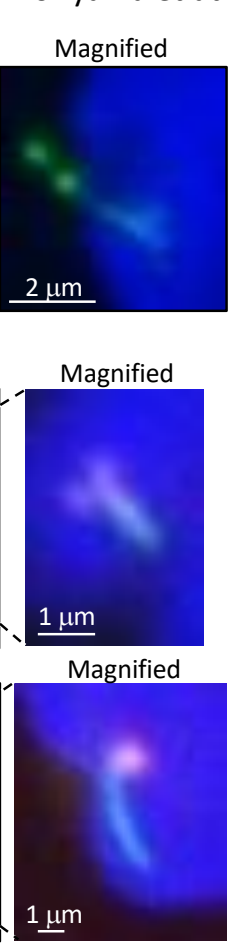

Figure 1. Human Primary immune cells have primary cilia-like structure.

(A) HE staining of healthy human skin. (B) Langerin-positive langerhans cells and acetylated tubulin (primary cilia marker) in human healthy epidermis. Arrowhead indicates primary cilia-like structure. Blue dot line indicates epidermal basal layer. White dot line indicates stratum corneum. Dot box indicates magnified area shown in right. (C) Immunostaining image of human PBMC stained with acetylated tubulin or glutamylated tubulin. Dot box indicates magnified area shown in right. (D) Immunostaining image of human PBMC expressing Arl13B-GFP. Cells were electroporated with Arl13B-GFP expression plasmid, then immunostained by using anti GFP antibody. Dot box area was magnified. (E) Human PBMC was cultured in media supplemented with $10 \%$ FBS (serum +) or $0.5 \%$ FBS (Serum -) for $16 \mathrm{hrs}$, then immunostained with acetylated tubulin (Green), and pericentrin (Red). Dot box area were magnified. (F) Number of ciliated cells or cilia length shown in (E). Bar indicates median. ${ }^{*} \mathrm{P}<0.05$. (Mann-Whitney $U$ test).

(G) Electron microscope image of primary cilia-like structure. Arrow head indicates ciliary vesicle like structure. Asterisk indicates goldi apparatus. Star indicates nucleus. Dot box indicates magnified area shown in right. (H) Monocyte, pDC and cDC were isolated from PBMC using flow cytometry, then isolated cells were immunostained with acetylated tubulin (green), and pericentrin (red). 


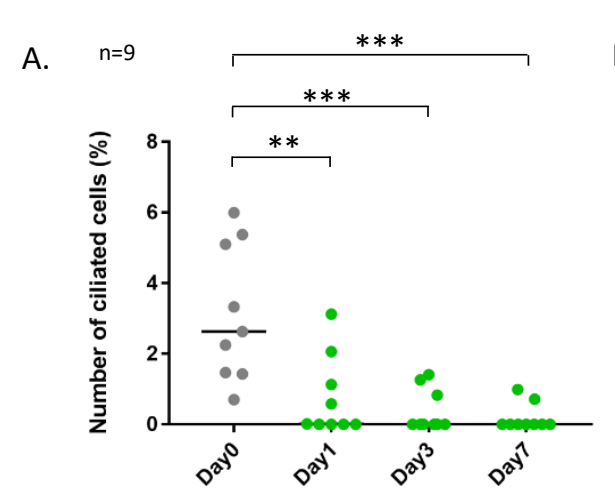

B. $n=12$

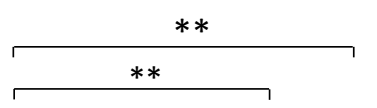

C. ${ }^{n}=18$

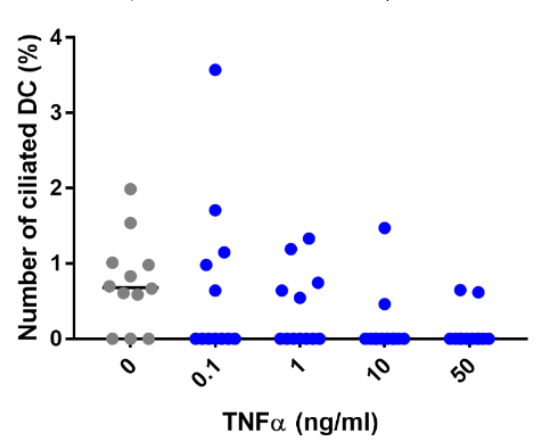

E.

D.

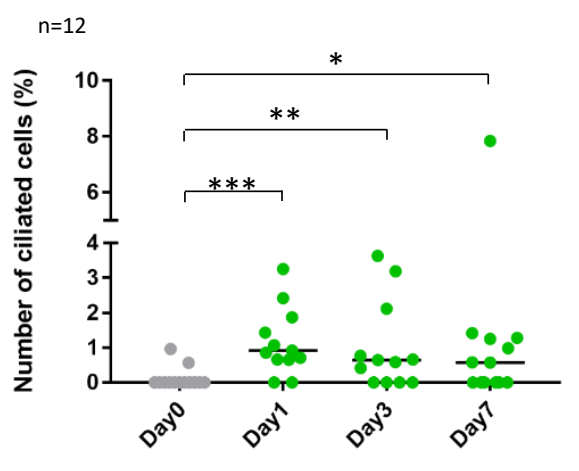

G.

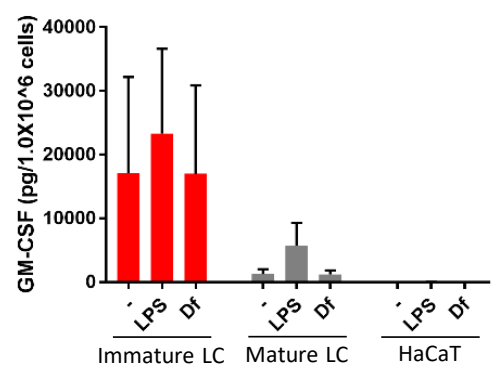

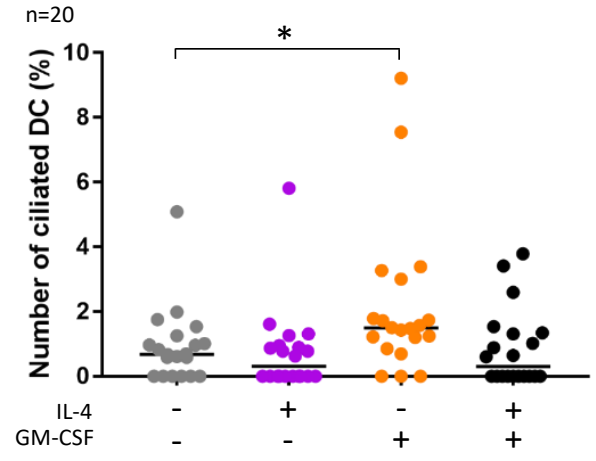

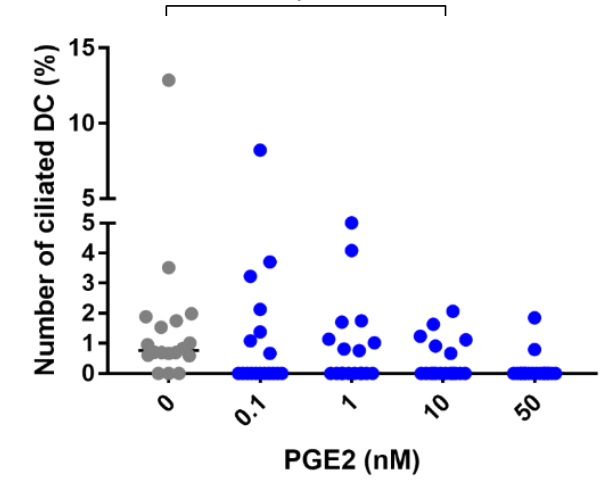

F.

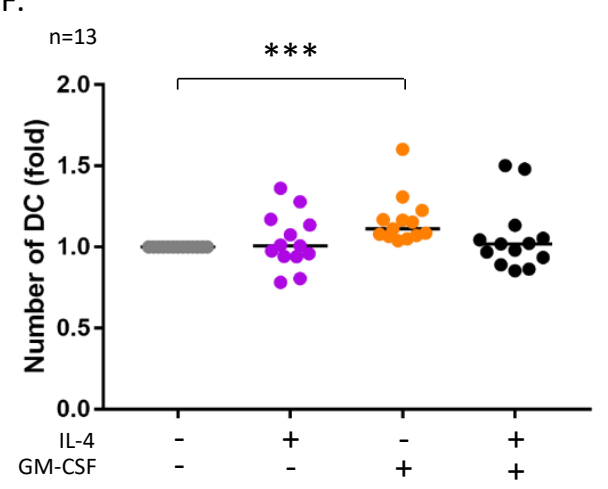

Figure 2. DC maturation decreases primary cilia.

(A) CD14+ monocytes were isolated from human PBMC by magnetic beads, then differentiated into DC by stimulating with $50 \mathrm{ng} / \mathrm{ml}$ $\mathrm{GM}-\mathrm{CSF}, 50 \mathrm{ng} / \mathrm{ml} \mathrm{IL-4}$ and $50 \mathrm{ng} / \mathrm{ml} \mathrm{TNF} \alpha$. Percentage of ciliated cell was shown in graph. Bar indicates median. ${ }^{* *} \mathrm{P}<0.01$, *** $P<0.001$ (Mann-Whitney U test). $n=9$. (B, C) Human DC isolated from PBMC was stimulated with (B) TNF $\alpha$, or (C) PGE2 for 24 hrs. Percentage of ciliated cells were shown in graph. Bar indicates median. ${ }^{*} P<0.05,{ }^{*} * P<0.01$ (Mann-Whitney $U$ test). $n=12$ and $n=18$, respectively. (D) CD14+ monocytes were isolated from human PBMC by magnetic beads, then differentiated into $\mathrm{DC}$ with $50 \mathrm{ng} / \mathrm{ml}$ GM-CSF and $50 \mathrm{ng} / \mathrm{ml} \mathrm{IL-4}$. Percentage of ciliated cell was shown in graph. Bar indicates median. ${ }^{*} \mathrm{P}<0.05,{ }^{*}{ }^{*} \mathrm{P}<0.01,{ }^{* * *} \mathrm{P}<0.001$. (Mann-Whitney U test). $n=12$. (E) DCs isolated from PBMC were cultured for $24 \mathrm{hrs}$ with $50 \mathrm{ng} / \mathrm{ml} \mathrm{IL-4}$ and $50 \mathrm{ng} / \mathrm{ml} \mathrm{GM-CSF}$. Percentage of ciliated cells was counted and graphed. Bar indicates median. ${ }^{*} P<0.05$, (Fisher's LSD). $n=20$. (F) DCs isolated from PBMC were cultured with $50 \mathrm{ng} / \mathrm{ml} \mathrm{IL-4,} 50 \mathrm{ng} / \mathrm{ml} \mathrm{GM-CSF}$ with CCK buffer for $48 \mathrm{hrs}$, then relative number of cells were calculated by measuring absorbance. Bar indicates median. ${ }^{* * *} \mathrm{P}<0.001$ (Mann-Whitney $\mathrm{U}$ test). $\mathrm{n}=13$. (G) Average of GM-CSF expression measured by ELISA. Immature LC, Mature LC and HaCaT cells were stimulated with $10 \mu \mathrm{g} / \mathrm{ml}$ LPS or $10 \mu \mathrm{g} / \mathrm{ml} \mathrm{Df}$ for $24 \mathrm{hrs}$ in media supplemented with $0.5 \%$ serum. Culture supernatant was used for assay. Error bar shows SEM. $\mathrm{n}=5$. 
Figure 3. Primary cilia regulates proliferation and maturation via PDGFR $\alpha$ signaling.

A.

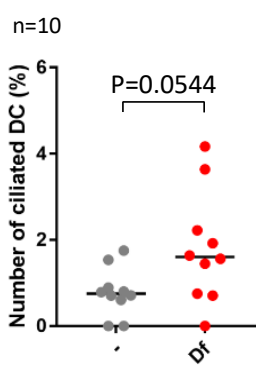

B.

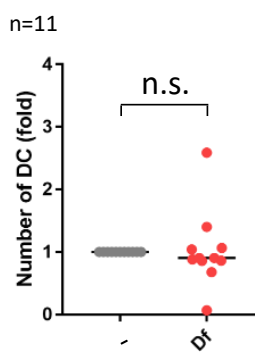

C. $n=15$

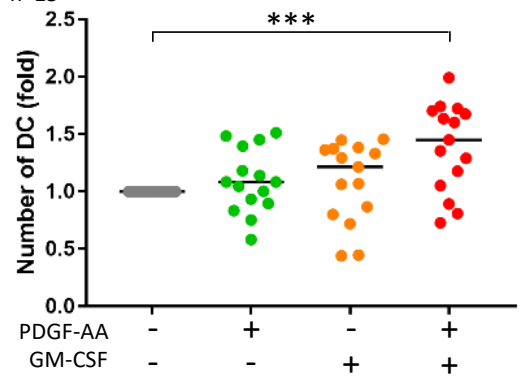

D.

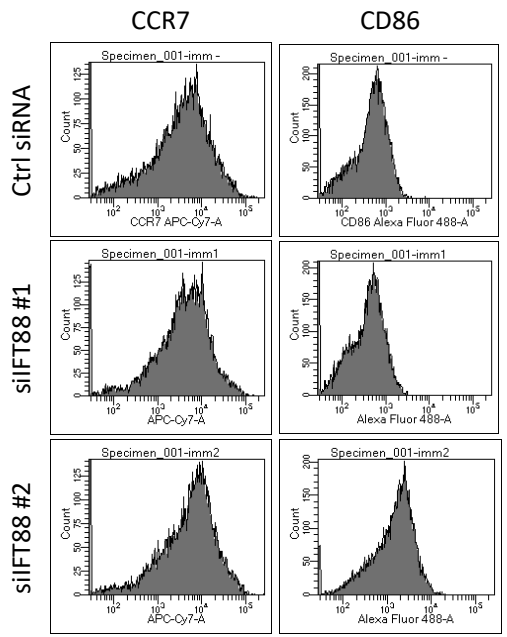

E.

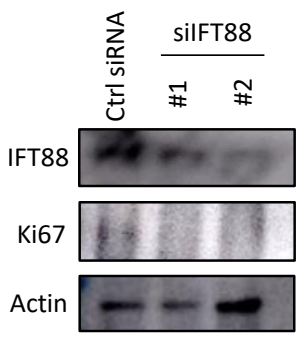

F.

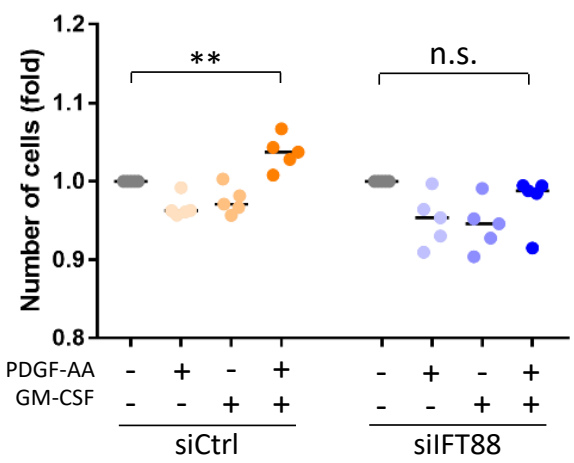

Figure 3. Primary cilia regulates proliferation and maturation via PDGFR $\alpha$ signaling.

(A) DCs were cultured with $1 \mu \mathrm{g} / \mathrm{ml}$ Df for $48 \mathrm{hrs}$, then counted ciliated cell number. Bar indicates median. ${ }^{* * P}<0.01$ (Mann-Whitney $U$ test). (B) Relative number of DC was measured $48 \mathrm{hrs}$ after stimulation with $1 \mu \mathrm{g} / \mathrm{ml}$ Df by measuring absorbance. Bar indicates median. ${ }^{*} \mathrm{P}<0.05$ (Mann-Whitney $\mathrm{U}$ test). (C) Relative number of DC was measured 48 hrs after stimulation with $10 \mathrm{ng} / \mathrm{ml}$ PDGF-AA or $10 \mathrm{ng} / \mathrm{ml}$ GM-CSF. For the control of PDGF-AA stimulation, PDGF-AA solvent (4 mM $\mathrm{HCl}, 0.1 \% \mathrm{BSA}$ ) was added. Bar indicates median. Fisher's LSD was performed. ${ }^{* *}{ }^{*} \mathrm{P}<0.001 \mathrm{n}=15$. (D) To induce immature $D C$, THP1 cells were cultured with $100 \mathrm{ng} / \mathrm{ml} \mathrm{GM-CSF}$ and $100 \mathrm{ng} / \mathrm{ml} \mathrm{IL-4}$ for 5 days. At day5, cells were electroporated with $100 \mathrm{nM}$ siRNA targeting IFT88 or control siRNA. Two days after electroporation, expression of cell marker was measured by flow cytometry. (E) IFT88 and Ki67 expression in THP1-derived immature DC was investigated by western blotting. (F) Immature DC derived from THP1 was electroporated with $100 \mathrm{nM}$ siRNA. Two days after electroporation, cells were stimulated with10 ng/ml PDGF-AA or $10 \mathrm{ng} / \mathrm{ml}$ GM-CSF for 48 hours. Proliferation activity was measured by measuring absorbance with adding CCK buffer. Cells were Bar indicates median. ${ }^{* *} \mathrm{P}<0.01$ (Student $\mathrm{T}$ test). 


\section{Figure 4. Primary cilia are increased in atopic dermatitis.}
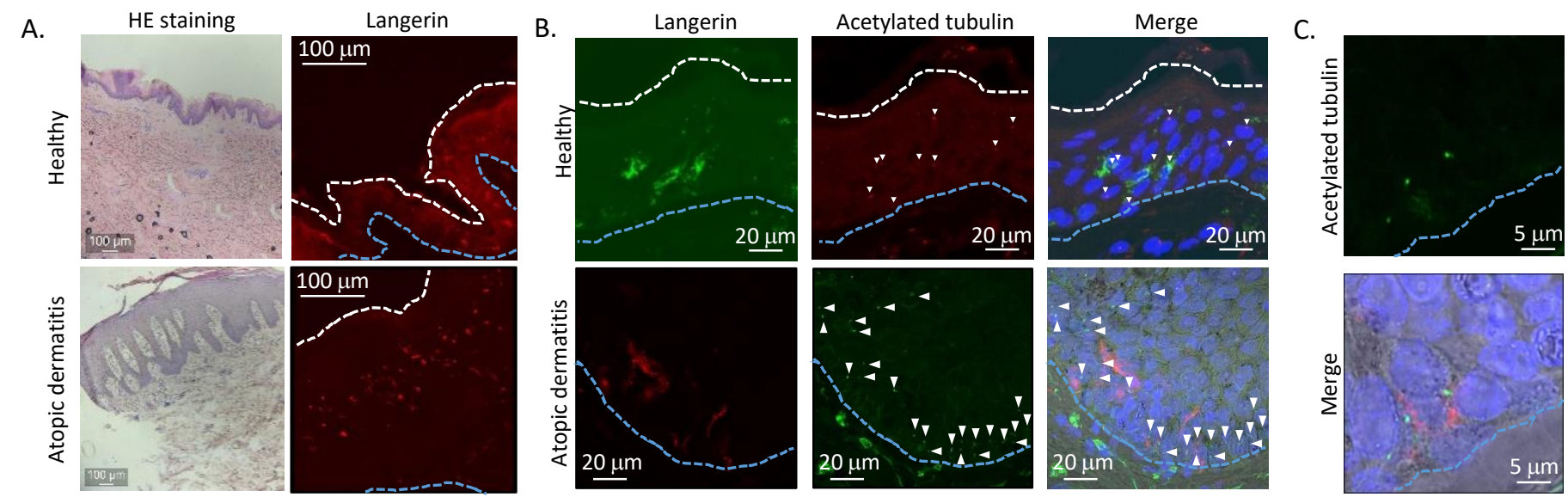

D.

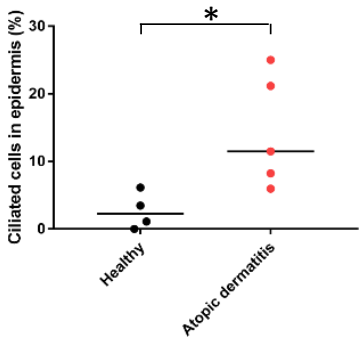

F.
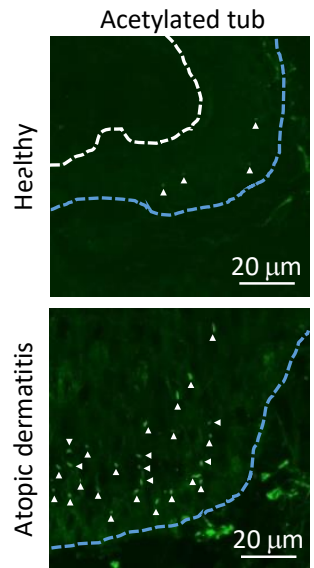
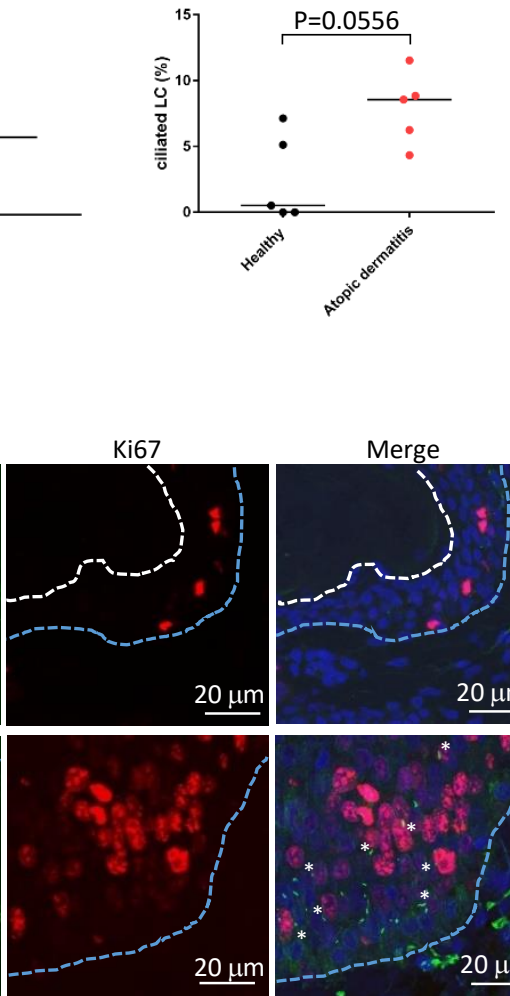

Merge

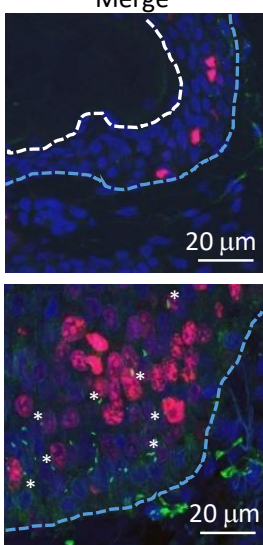

E.

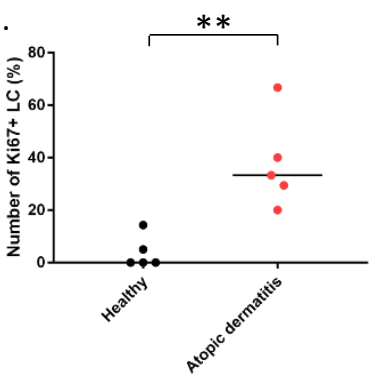

G.
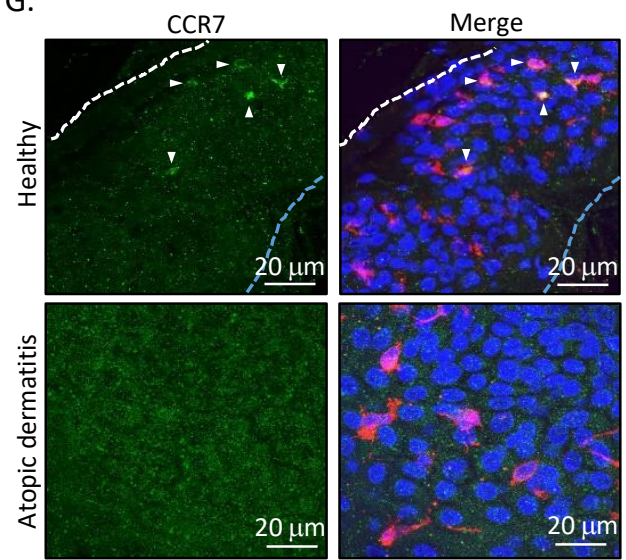

Figure 4. Primary cilia are increased in atopic dermatitis.

(A) HE staining of human skin and Immunostaining of langerin in epidermis. Blue dot line shows basal layer. White dot line shows stratum corneum. (B) Langerin and acetylated tubulin staining in human epidermis. Blue dot line shows basal layer. Arrowhead indicates primary cilia-like structure. (C) Magnified image of ciliated LC in atopic skin. Langerin is shown in red. (D) Percentage of ciliated epidermal cells (upper), and ciliated LC (lower) in healthy or atopic dermatitis epidermis. Healthy samples; $n=4$ or $n=5$, atopic skin sample; $n=5$. Bar indicates median. ${ }^{*} P<0.05$ (Mann-Whitney $U$ test). (E) ki67-positive $L C$ in healthy and atopic skin were counted and graphed. ${ }^{*} \mathrm{P}<0.05$ (Mann-Whitney $U$ test). (F) Immunostaining for acetylated tubulin (green), and ki67 (red) in epidermis from healthy donor or atopic dermatitis patient. Blue dot line shows basal layer. White dot line shows stratum corneum. Arrow head shows primary cilia. Asterisk shows ki67-positive ciliated cells. (G) Immunostaining for CCR7 (green), and langerin (red) in epidermis. Arrow head indicates CCR7 positive LC 


\section{Figure 5. Keratinocyte differentiation marker, Loricrin expression correlates with primary cilia rate}

A.
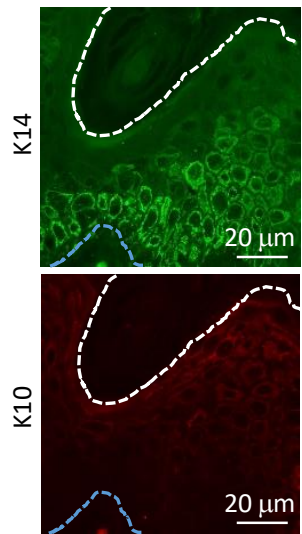

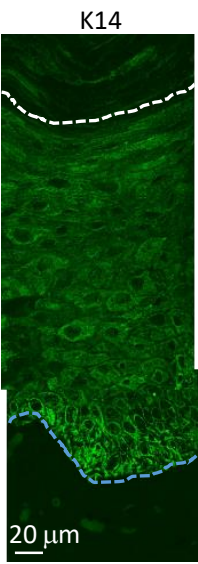

K10

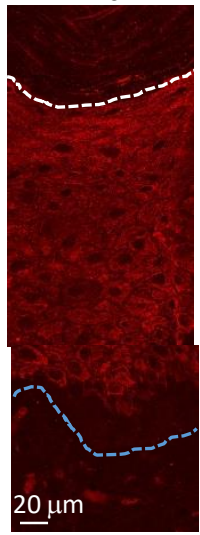

C.

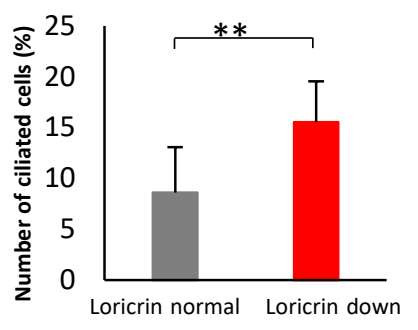

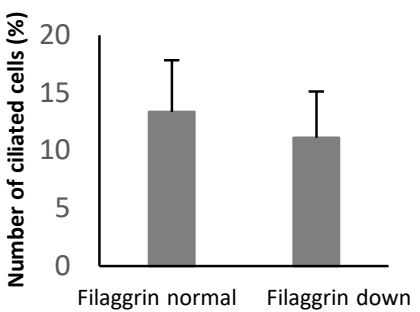

D.

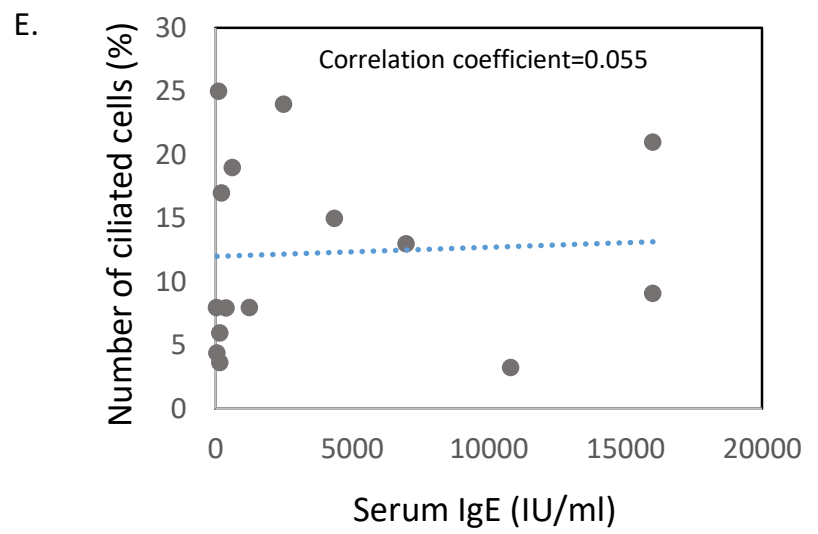

- Loricrin down $\quad$ L Loricrin normal

- IgE high - IgE low

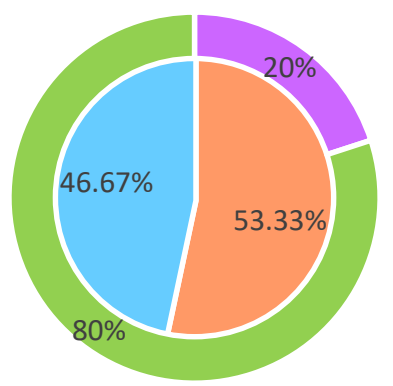

Figure 5. Keratinocyte differentiation marker, Loricrin expression correlates with primary cilia rate

$(A, B)$ Immunostaining for K14 (green) and K10 (red) in (A) healthy epidermis, and in (B) atopic epidermis. (C) Correlation between primary cilia and epidermal barrier Protein, loricrin and Filaggrin. Atopic dermatitis epidermis were immunostained with Loricrin or Filaggrin, with acetylated tubulin, then calculated percentage of ciliated cells. (D) Percentage of atopic dermatitis patients showing Loricrin normal, loricrin down, IgE low, IgE high, respectively. (E) Correlation between primary cilia percentage and serum IgE level in atopic dermatitis patients. 